Waheed, W., Hussain, N. \& Creed, F. (2003)

Psychiatric services for ethnic minority groups: a third way (letter)? British Journal of Psychiatry, 183, 562-563.

K. Bhui Barts \& The London School of Medicine, Queen Mary, London El 4NS, UK

\section{Dhat syndrome: a functional somatic syndrome?}

We read with interest the historical overview on dhat syndrome by Sumathipala $e t$ al (2004). We agree with the authors' contention that categorising it as a culturebound syndrome is not likely to advance research. The authors examine the nosological significance of this disorder and suggest the possibility of culturally influenced somatoform disorder, although they do not offer a detailed model. In the spirit of Sumathipala et al's conclusion that there are no absolute truths when it comes to classificatory systems, we propose the following formulation.

Fatigue is a common symptom in dhat syndrome (Bhatia \& Malik, 1991). Disorders with fatigue as the main symptom are often grouped together as functional somatic syndromes (Barsky \& Borus, 1999). The basic cognitive formulation offered to explain these disorders is based on somatosensory amplification, misattribution and abnormal illness behaviour. We have incorporated societal and cultural factors along the lines of the socio-somatic model (Kirmayer \& Young, 1998) to explain dhat syndrome as a functional somatic syndrome.

In cultures where open discussion about sexual issues is taboo and fears about masturbation exist, the urogenital system is likely to be the focus of preoccupation. Under stress, persons predisposed to amplification of somatic symptoms and health anxiety may focus attention on physiological changes such as turbidity of urine and tiredness, and misattribute them to loss of semen in the light of widely prevalent health beliefs. These beliefs may then be confirmed by friends and other lay sources as well as by local practitioners subscribing to similar models.

We have recently completed a study showing significantly higher scores on measures of amplification, hypochondriacal beliefs and abnormal illness behaviour in patients with dhat syndrome compared with medical controls. The above model needs to be examined further in both quantitative and qualitative studies. The practical implication of this formulation is that it suggests a viable treatment model based on psychoeducation and culturally informed cognitive-behavioural therapy, which has been demonstrated to be feasible in the Indian subcontinent (Sumathipala $e t$ al, 2000).

Barsky, A. J. \& Borus, J. F. (1999) Functional somatic syndromes. Annals of Internal Medicine, 130, 910-921.

Bhatia, M. S. \& Malik, S. C. (199I) Dhat syndrome - a useful diagnostic entity in Indian culture. British journal of Psychiatry, 159, 691-695.

Kirmayer, L. J. \& Young, A. (1998) Culture and somatization: clinical, epidemiological, and ethnographic perspectives. Psychosomatic Medicine, 60, 420-430.

Sumathipala, A., Hewege, S., Hanwella, R., et al (2000) Randomized controlled trial of cognitive behaviour therapy for repeated consultations for medically unexplained complaints: a feasibility study in Sri Lanka. Psychological Medicine, 60, 420-430.

Sumathipala, A., Siribaddana, S. H. \& Bhugra, D. (2004) Culture-bound syndromes: the story of dhat syndrome. British Journal of Psychiatry, 184, 200-209.

G. Ranjith South London and Maudsley NHS Trust, Norwood CMHT, 19-2I Knights Hill, London SE27 OHS, UK

R. Mohan Health Service Research Department, Institute of Psychiatry, London, UK

\section{Jaspers' concept of primary delusion}

Jaspers has appeared recently in the pages of the Journal both to support the impossibility of studying psychopathology scientifically (Turner, 2003) and to defend the notion of a delusion arising as a consequence of the modularisation of a nonmodular belief system, linked to dopamine dysfunction (Jones, in Jones et al, 2003), and thus of a scientific psychopathology. Jaspers has also been cited as an outmoded influence on psychopathological thinking, which should now be discarded (Delespaul \& van Os, in Jones et al, 2003). Such a disagreement may hinge upon Jaspers' conception of a primary delusion.

For Jaspers the primary delusion has two elements. First, there is a radical change in subjectivity: 'We observe that a new world has come into being' (Jaspers, 1963: p. 284). Such a new world is more than the presence of a false belief, it is a transformation of experience as a whole. Second, there is the element of meaning: 'All primary experience of delusion is an experience of meaning' (Jaspers, 1963: p. 103). 'The experiences of primary delusion are analogous to this seeing of meaning, but the awareness of meaning undergoes a radical transformation' (Jaspers, 1963: p. 99).

Jones, drawing on Campbell's work on delusions (Campbell, 2002), wishes to recruit Jaspers as a rationalist. This is the concept that pathological top-down mechanisms can render delusions explicable. Portraying Jaspers thus misrepresents his position, as the conception he offers us of primary delusion is one of a new state of consciousness, and this may be as resistant to mechanistic explanation as is normal consciousness (the so-called 'hard problem'; Chalmers, 1996). In this respect, Jaspers may be better thought of as a 'mysterian’ (McGinn, 1993). Mysterians hold that although consciousness is biologically mediated, it is inexplicable mechanistically. Nowhere in the General Psychopathology does Jaspers discuss the mechanism of primary delusion and he explicitly rejects a modular conception of psychopathology, as envisaged by his contemporary Wernicke: 'As soon as this theory is transferred to everything psychic as if it were analogous it ceases to further our knowledge' (Jaspers, 1963: p. 537). The existence of primary delusion is left as an 'ununderstandable' fact.

Delespaul \& van Os want to discard the concept of primary delusion. In doing so they address only what Jaspers terms 'delusion-like ideas'. Jaspers would have had no difficulty regarding these on a continuum with normal beliefs and it is by virtue of this that he regarded them as understandable. Primary delusions are left untouched on this account and yet they remain central to the clinical experience of major psychosis - the radical and sometimes rapid transformation from a given way of perceiving, thinking, affecting, acting to another, which colours all of subjective experience.

We agree that progress in psychopathology is dependent upon overcoming Jaspers' pessimism about understanding primary delusion. How this can be achieved remains an open question but progress may not come if we misrepresent Jaspers' great contribution.

Campbell, J. (2002) Rationality, meaning and the analysis of delusion. Philosophy, Psychiatry and Psychology, 8, 89-100.

Chalmers, D. (1996) The Conscious Mind. Oxford: Oxford University Press.

Jones, H./Delespaul, P. \& van Os, J. (2003) Jaspers was right after all - delusions are distinct from normal beliefs (debate). British Journal of Psychiatry, 183 285-286. 